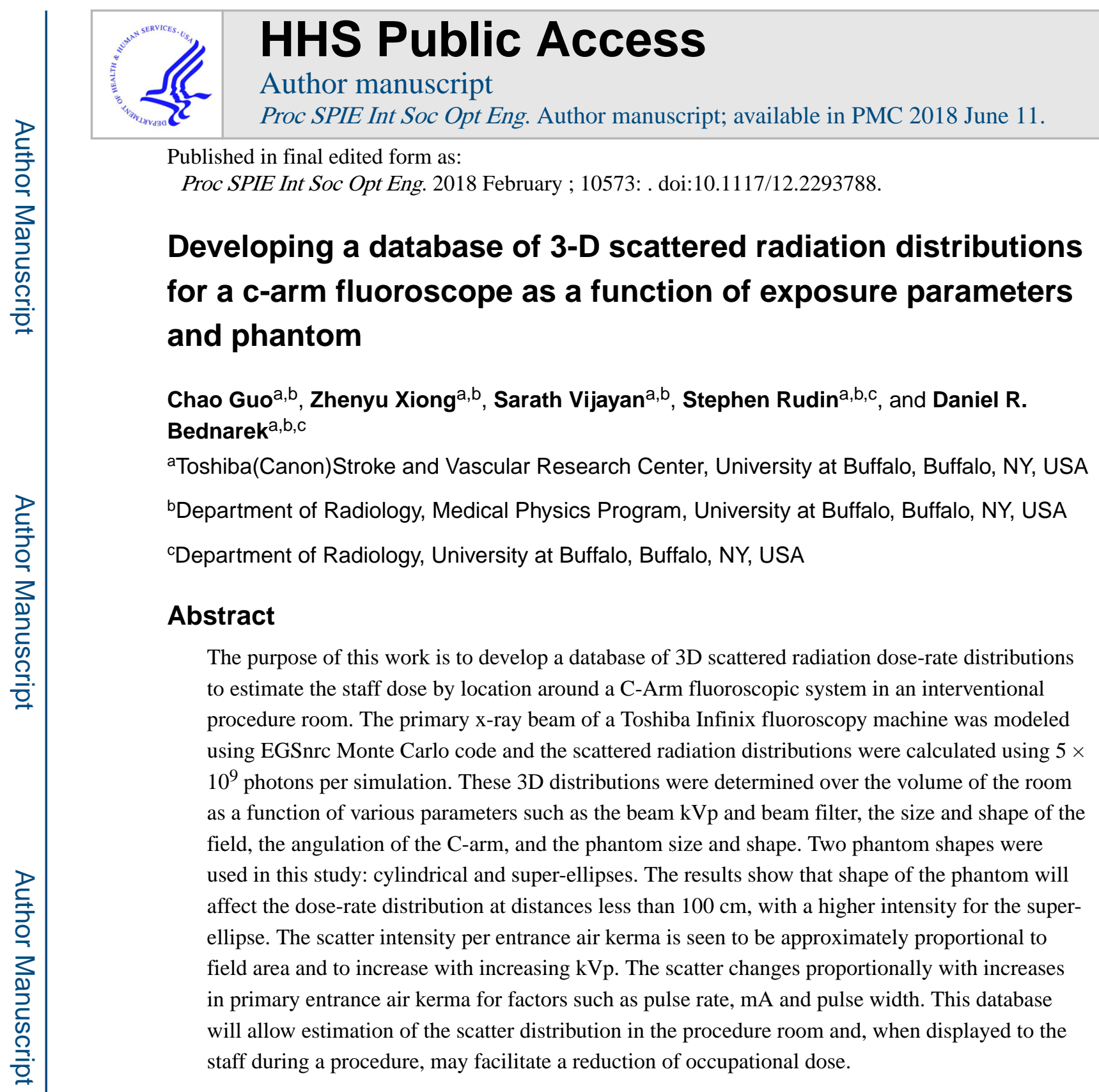

\title{
1: INTRODUCTION
}

In addition to the potential for significant dose to the patient's skin, fluoroscopically-guided interventional procedures can result in high dose to staff in the procedure room due to scattered radiation. To predict the scattered radiation to staff during fluoroscopically-guided interventions, the scatter distribution must be known as the exposure parameters are changed during a procedure. The purpose of this investigation is to determine how the scatter distribution in the room changes with beam energy, field size, beam angulation, and the shape and size of the "patient" and to develop a database as a function of these parameters. This database can then be used to estimate the scatter level throughout the room during a dynamic interventional procedure when these parameters are known. 


\section{2: METHODS AND MATERIALS}

EGSnrc Monte Carlo simulation software was used to calculate the room scattered radiation distribution with different beam energy and field size change, as well as different shape and size of the phantom.

All calculations used $5 \times 10^{9}$ photons per simulation and all simulations were run at the Center for Computational Research of the University at Buffalo. Every simulation is split into 10 parallel processing cores to boost the running speed.

Two different phantom shapes were simulated: a round $30 \mathrm{~cm}$ diameter cylinder and superellipses (SE), which are essentially rectangles with rounded corners, to better approximate the shape of a patient torso as shown in figure 1. The width of the super ellipse for the results shown here is $30.75 \mathrm{~cm}(\mathrm{w}=0.64 * \mathrm{BMI}+17.95 \text { with a BMI of } 20)^{1}$ and the thickness is $20 \mathrm{~cm}$. Fig. 2 illustrates the exposure setup for the phantom and the vertical plane for which the scatter distributions are shown in this paper. For this study, all phantoms were water equivalent.

\section{Validation Setup}

Validation measurements were performed to ensure the EGSnrc Monte Carlo simulated results agree with the actual scatter distributions. The validation setup is shown in figure 3 . All measurements used a square water equivalent phantom with dimension of $30 \mathrm{~cm} \times 30$ $\mathrm{cm} \times 28 \mathrm{~cm}$ high placed on the $\mathrm{C}$-arm table. The beam was directed horizontally to avoid the scattering from the table. Two $6 \mathrm{cc}$ ion chambers are used to measure the entrance dose and the scattered dose simultaneously. The scatter dose was measured in two directions on a plane parallel to the side of the phantom; one direction was parallel to the beam direction and the other perpendicular to the beam direction. For the parallel direction, a range from -4 $\mathrm{cm}$ to $40 \mathrm{~cm}$ are measured with an increment of $4 \mathrm{~cm}$. The 0 point is located at the center of the phantom. For the perpendicular direction, a range from $-5 \mathrm{~cm}$ to $20 \mathrm{~cm}$ are measured with an increment of $5 \mathrm{~cm}$. These measurements are made at two distances from the phantom: $15 \mathrm{~cm}$ from the surface and $35 \mathrm{~cm}$ from the surface. The exposure time was set to 30 seconds, the tube voltage to $120 \mathrm{kVp}$, the tube current at $200 \mathrm{~mA}$ and the frame rate was 15 frames per second.

\section{3: RESULTS AND DISCUSSION}

\section{Different entrance field size}

Figure 4 shows scatter distributions for a vertical beam aiming upwards with the central axis at the center of the cylindrical phantom; the distributions are shown for a vertical plane at the indicated distance from, and parallel to, the centerline of the phantom. For these figures, the scatter values are given in units of mGy scatter air kerma per Gy entrance air kerma to the phantom. If the intensity were normalized to field area as shown in Figure 5, the shape of the scattering distributions are almost identical for different field size under the $120 \mathrm{kVp}$, with the larger the field size having the higher intensity. This could be find after normalized the highest intensity to the same value and compare with different field size as shows below. This means that the scatter value could be calculated for different field sizes if needed. 


\section{Different Shape of the phantom: Super ellipse (SE) and $30 \mathrm{~cm}$ cylindrical water phantom}

Figure 6 shows scatter distributions for a vertical beam aiming upwards with the central axis at the center of the phantom; the distributions are shown for a vertical plane at the indicated distance from, and parallel to, the centerline of the phantom. For these figures, the scatter values are given in units of mGy scatter air kerma per Gy entrance air kerma to the phantom. As we can see, the shape of the scattering changes with different shape of the phantom, especially at close distances. At larger distances, the intensity follows the inverse square law and the shape of the scattering distributions are similar provides a reference for the location of the phantom, which is not in the plane shown but at the same height. Please notice that the color scale changes between the $50 \mathrm{~cm}$ to $100 \mathrm{~cm}$ plots.

\section{Different energy: SE phantom}

Figure 7 shows scatter distributions for a vertical beam aiming upwards with the central axis at the center of the SE phantom; the distributions are shown for a vertical plane at the indicated distance from, and parallel to, the centerline of the phantom. For these figures, the scatter values are given in units of mGy scatter air kerma per Gy entrance air kerma to the phantom. According to these figures, we can see that the lower and higher energy will produce almost the same shape of the scatter distribution at the bottom on the side towards the tube. Greater differences are on the detector side; higher energy has greater penetration and the scatter distribution will have a peak build up along the beam direction toward the detector side.

\section{Validation}

Figure 8 shows validation results in a plane $35 \mathrm{~cm}$ from the phantom surface, comparing the Monte-Carlo calculation to the ionization chamber measurements at $120 \mathrm{kVp}$. The measurements made at different distances and at different energies are not shown but had less difference between measured and calculated data. The Monte Carlo simulated results are close to the measured result. Greater differences are in the $-5 \mathrm{~cm}$ to $5 \mathrm{~cm}$ and over $25 \mathrm{~cm}$ distance range. For the $-5 \mathrm{~cm}$ to $5 \mathrm{~cm}$ distances, secondary scattering from the table may increase the measured values. For the region over $25 \mathrm{~cm}$, the difference may be due to the angular dependence of the ion chamber which decreases the chamber response.

\section{4: CONCLUSIONS}

The simulated results show a large change in the scatter distribution for changes in exposure parameters, and phantom shape, especially near the phantom. Knowing how the scatter distribution changes with these factors and tracking these changes during a procedure, through a system such as the DTS, ${ }^{2}$ can provide real-time guidance in the reduction of staff dose. The validation results show that EGSnrc Monte-Carlo software can be used to accurately calculate the scatter dose distributions needed to create a database used for such a system.

\section{Acknowledgments}

This work was partially supported by Toshiba Medical Systems Corp. (Canon Group). The resources of the Center for Computational Research of the University at Buffalo were used for the Monte-Carlo calculations. 
DISCLOSURES

The authors receive research support from Toshiba Medical Systems. The dose tracking system (DTS) software is licensed to Toshiba Medical Systems by the Office of Science, Technology Transfer and Economic Outreach of the University at Buffalo.

\section{References}

1. Bordier $\mathrm{C}$, et al. Patient dose map indications on interventional $\mathrm{X}$-ray systems and validation with Gafchromic XR-RV3 film. Radiation Protection Dosimetry. Jun 17.2014

2. Rana V, et al. A tracking system to calculate patient skin dose in real-time during neurointerventional procedures using a biplane x-ray imaging system. Medical physics. 43(9):51315144. 


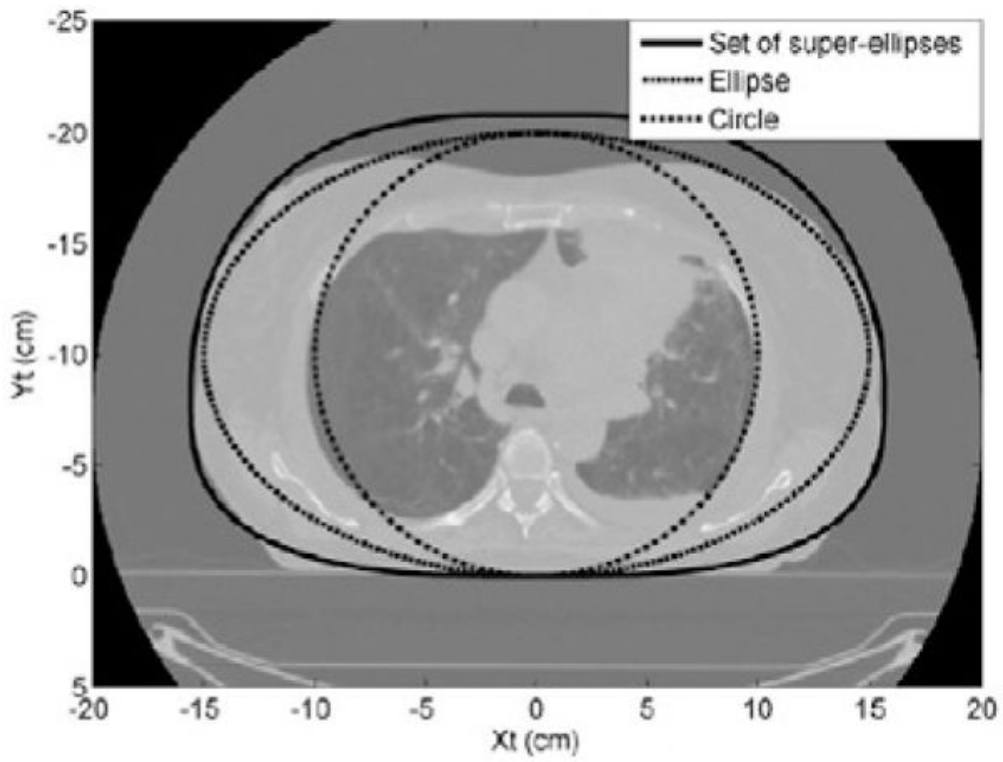

Fig. 1.

Scan patient compared with a set of super-ellipses (solid line), an ellipse (dashed line) and a circle (dash-dot line). ${ }^{1}$ 


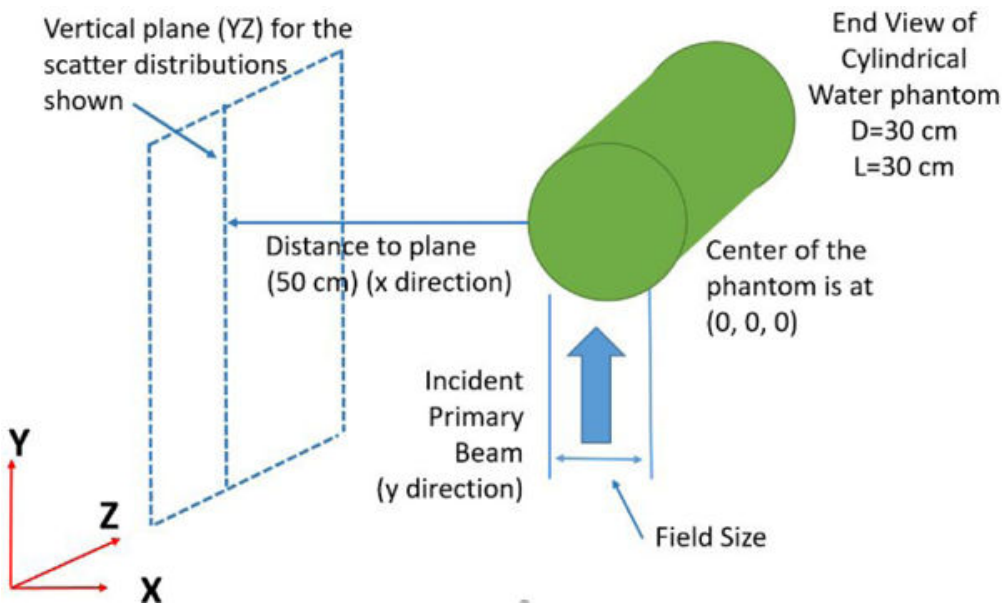

Fig. 2.

The setup for the phantom and the location of the vertical plane for the scatter distributions that are shown in this paper. This vertical plane is parallel to the axis of the "patient" (in and out of the screen) and at distances from the patient where the physician typically stands during the intervention. All results shown here are for a beam directed upwards from floor to ceiling. 


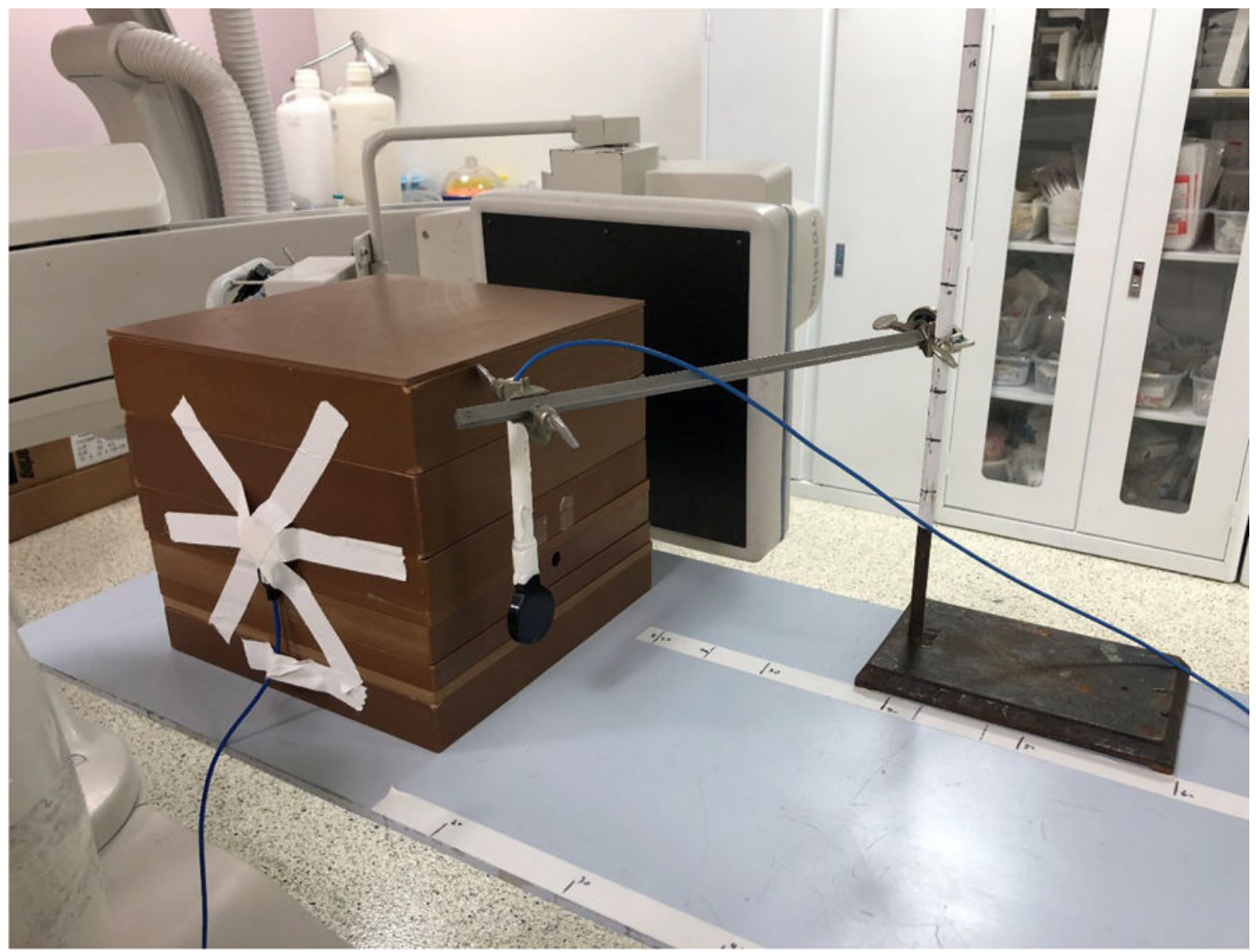

Figure 3.

Validation setup. One ionization chamber is taped to the entrance surface and a second is moved in a plane parallel to the lateral surface. 


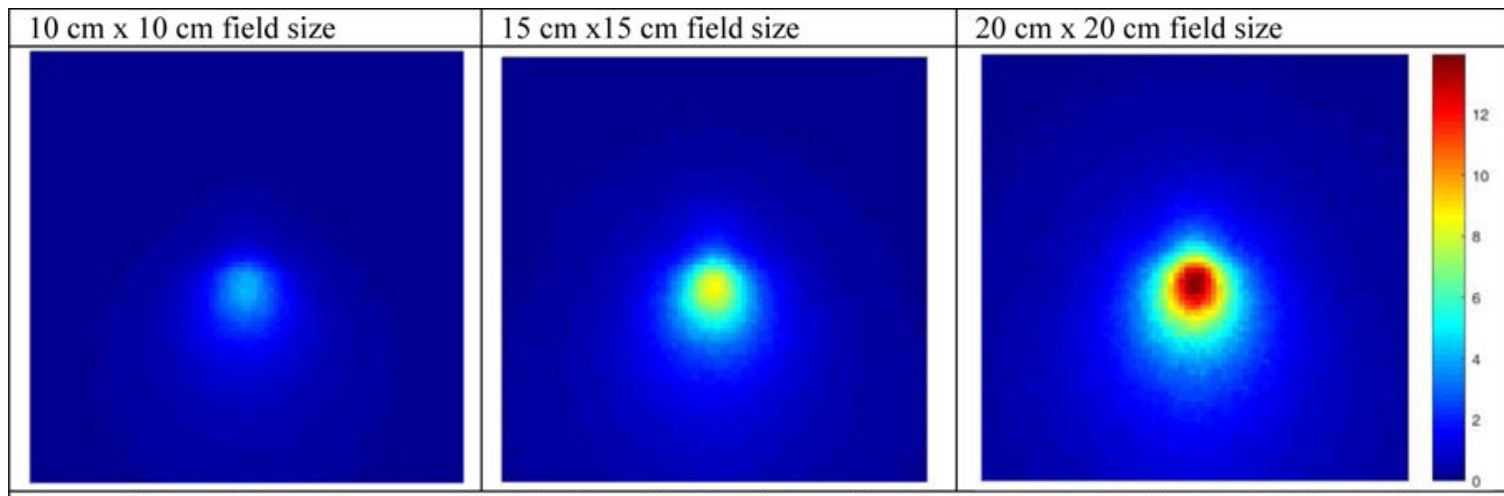

Lower figures: Upper plots normalized by the ratio of peak intensity of the $20 \times 20 \mathrm{~cm}$ field to the peak intensities of the $10 \times 10 \mathrm{~cm}$ and $15 \times 15 \mathrm{~cm}$ fields, respectively.

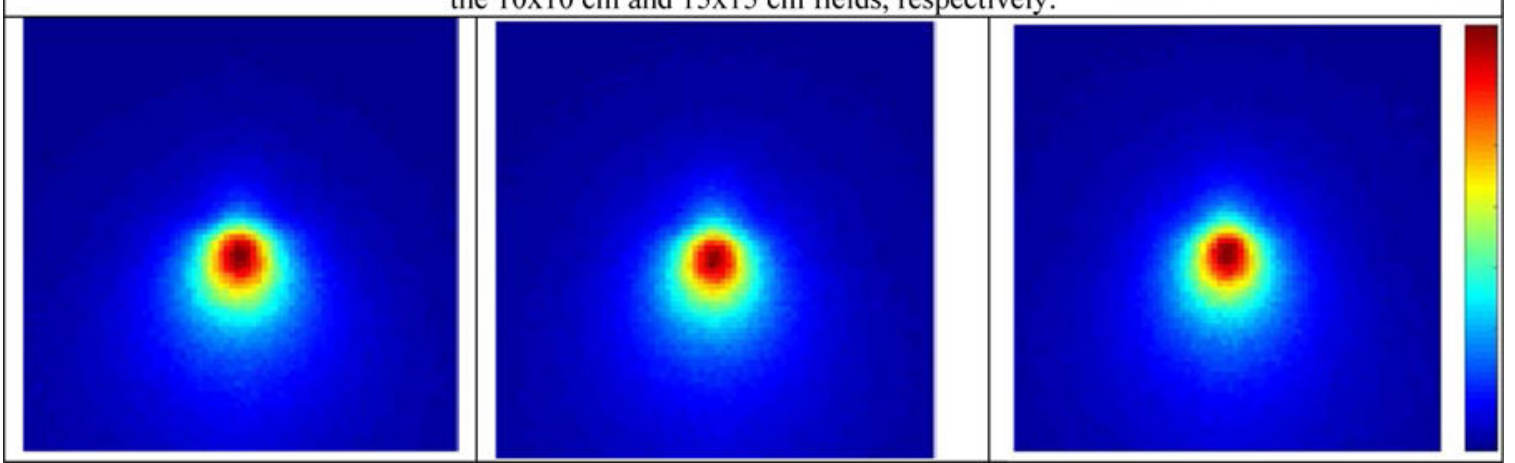

Fig. 4.

Upper: Color-coded scatter distribution in mGy scatter per Gy entrance air kerma in a vertical plane $50 \mathrm{~cm}$ from the center of a $30 \mathrm{~cm}$ cylindrical water phantom for different field sizes $(5 \mathrm{~cm}$ voxel size) at $120 \mathrm{kV}$. Lower: Scatter distributions normalized to the $20 \times 20 \mathrm{~cm}$ field peak intensity. Intensity varies approximately proportionally with field area. 

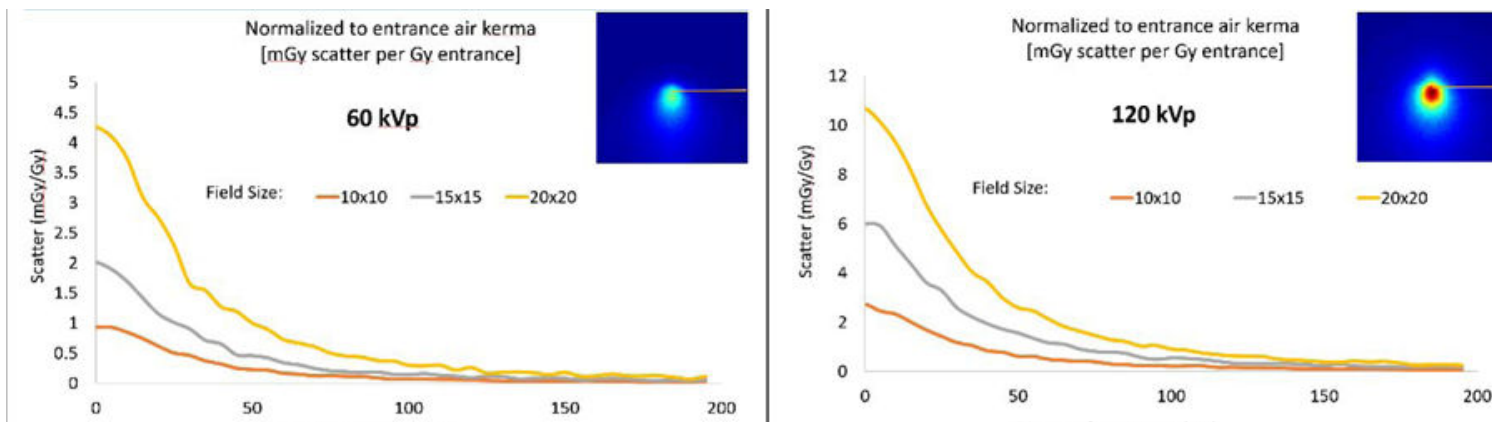

Distance from center $(\mathrm{cm})$

Normalized to entrance air kerma

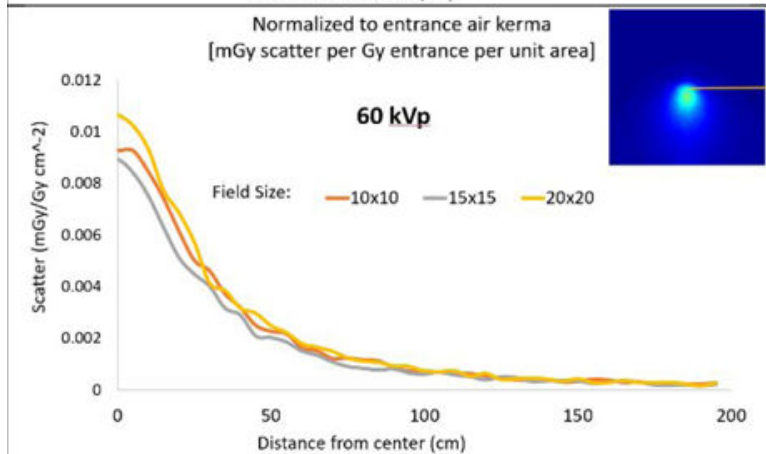

苋 4 4

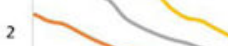

0

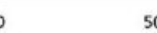

$50 \quad 100$ Distance from center $(\mathrm{cm})$

Normalized to entrance air kerma [mGy scatter per Gy entrance per unit area]
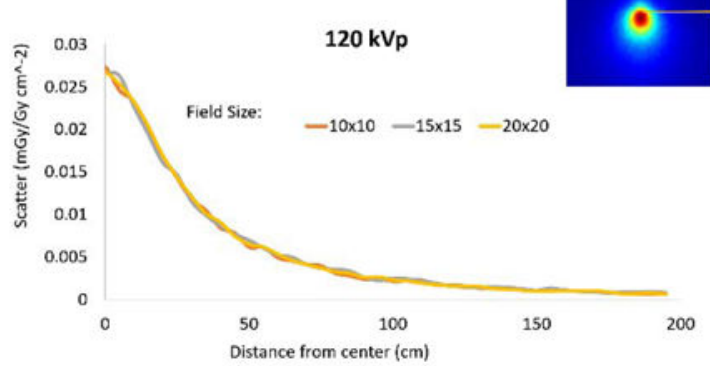

Fig 5.

Horizontal profile in vertical plane at $50 \mathrm{~cm}$. Plots on the top are the data before normalized to the field size. Plots on the bottom are nomolized data. 


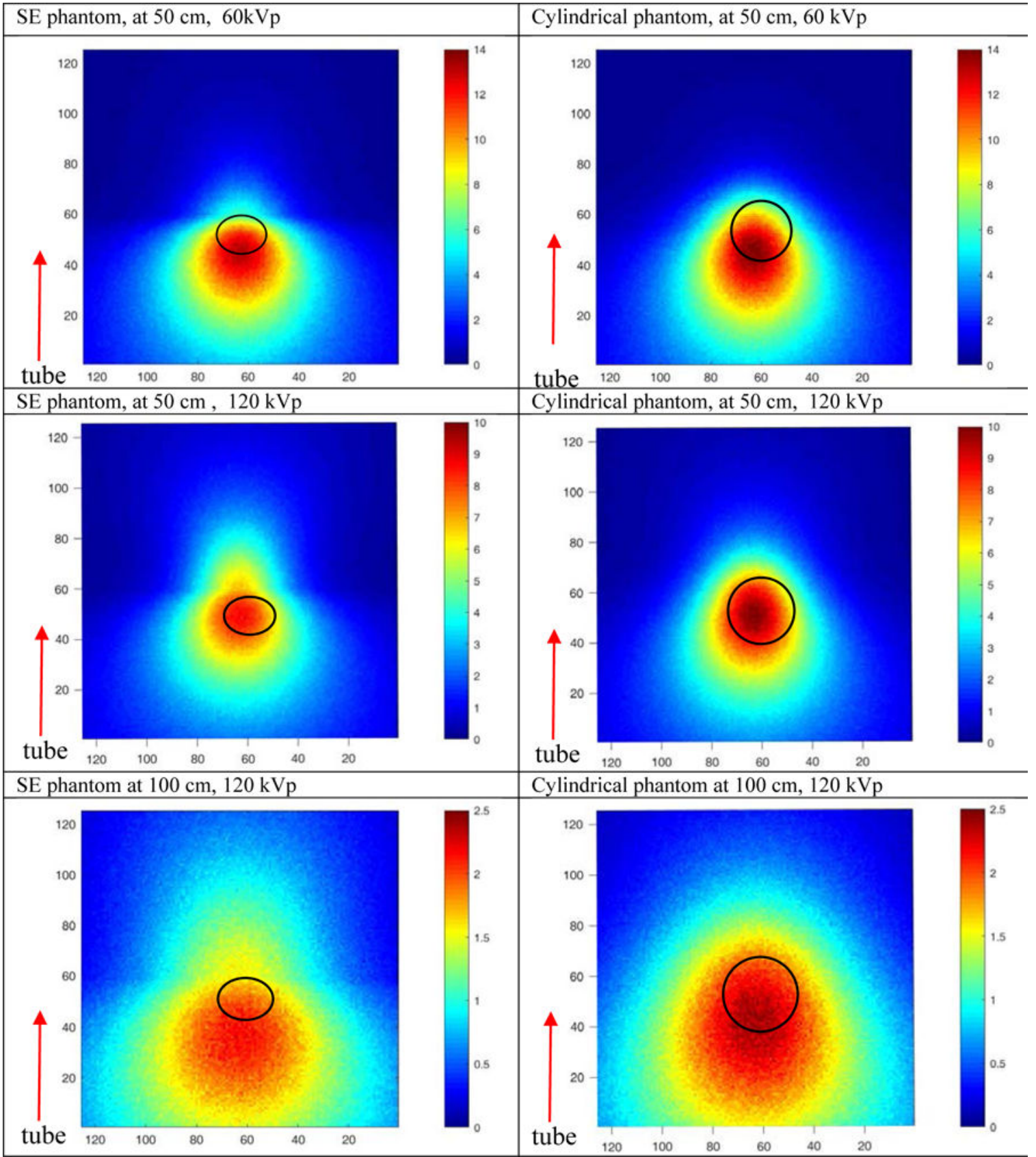

Fig. 6.

Comparison of scatter dose distributions between the SE phantom and $30 \mathrm{~cm}$ diameter cylindrical water phantom. The field size is $20 \mathrm{~cm}$ by $20 \mathrm{~cm}$ and the voxel size is $2 \mathrm{~cm}$. The shapes of the scattering dose distributions are close between the two phantoms but the intensity is somewhat higher for the cylindrical phantom at the center. The dose rate drops less than the inverse square law between $50 \mathrm{~cm}$ and $100 \mathrm{~cm}$ from the phantom. The black circle/ellipse 


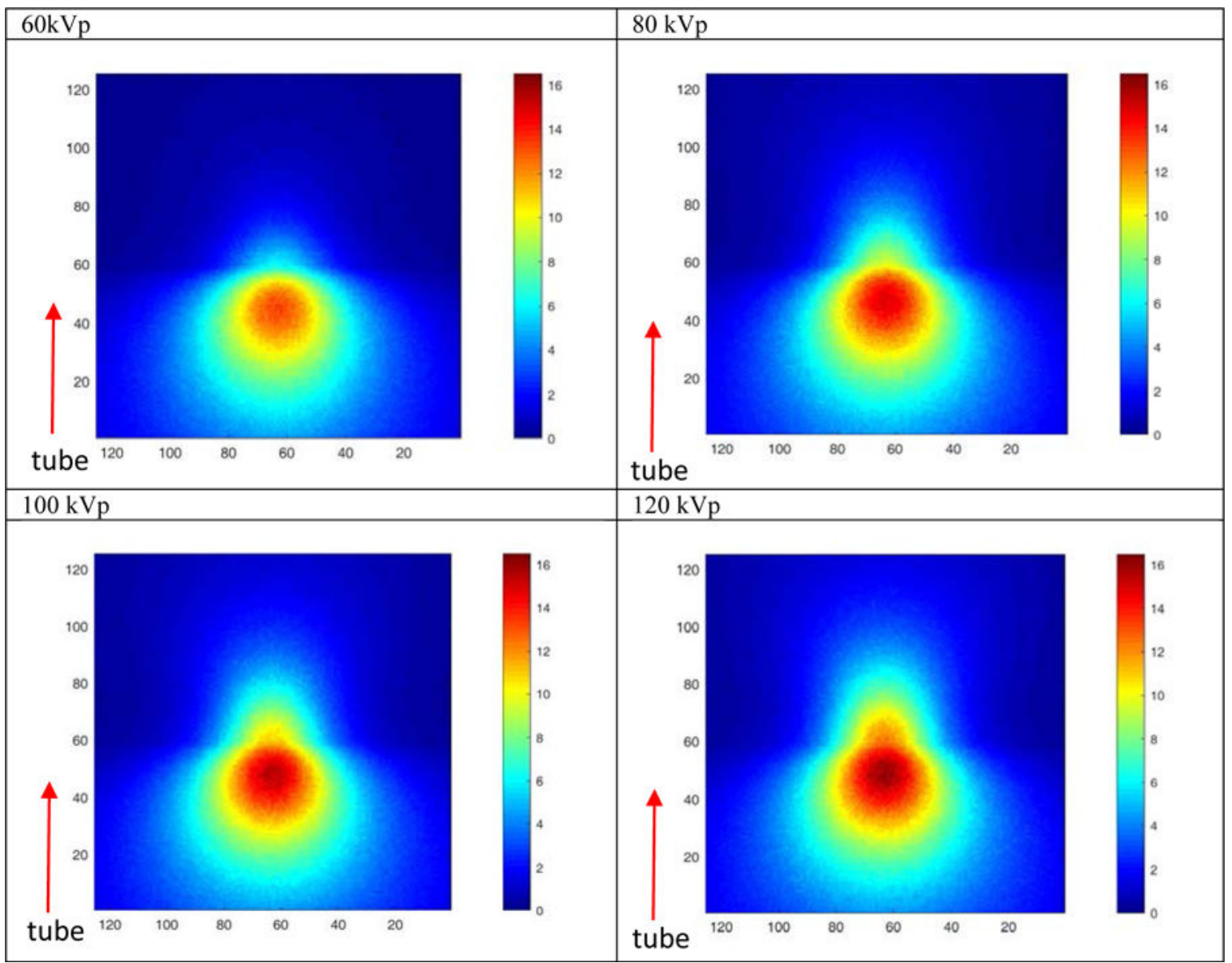

Fig.7.

Scatter distributions for the SE phantom at different energies in $\mathrm{mGy}$ per Gy entrance air kerma. Field size is $20 \mathrm{~cm} \times 20 \mathrm{~cm}$ and distance to the beam axis is $50 \mathrm{~cm}$. The scatter per incident air kerma increases with increasing $\mathrm{kVp}$. Please notice that the color scale of the figures is different compare to figure 6 . 


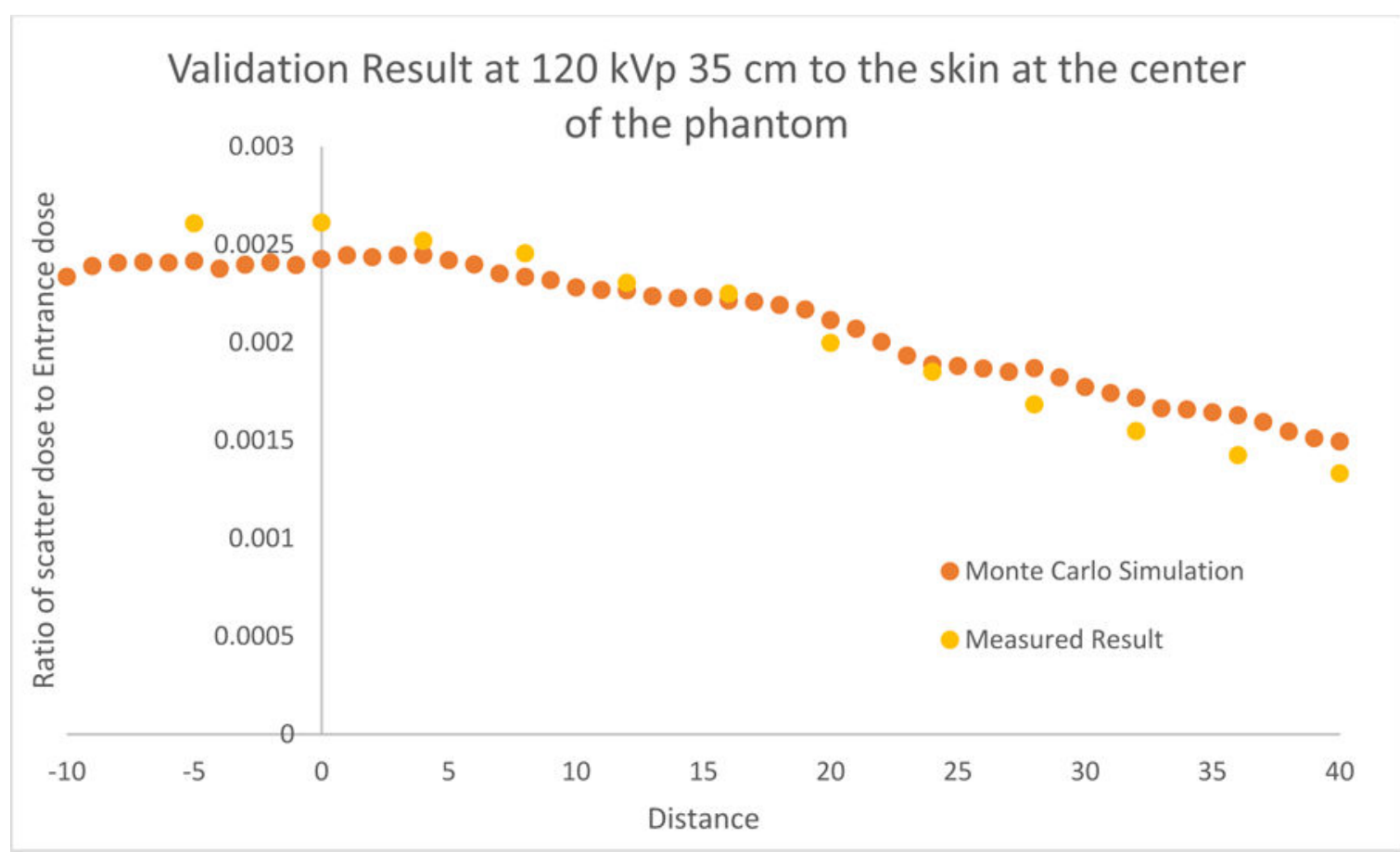

Figure 8.

Validation result at $120 \mathrm{kVp}, 35 \mathrm{~cm}$ from the phantom surface in a line perpendicular to the beam direction. 